\title{
Distribution of Mass and Energy in Five General Cosmic Models
}

\author{
Fadel A. Bukhari \\ Department of Astronomy, Faculty of Science, King Abdulaziz University, Jeddah, KSA \\ Email: fdbukhari@gmail.com
}

Received 3 March 2015; accepted 17 March 2015; published 20 March 2015

Copyright (C) 2015 by author and Scientific Research Publishing Inc.

This work is licensed under the Creative Commons Attribution International License (CC BY). http://creativecommons.org/licenses/by/4.0/

c) (i) Open Access

\begin{abstract}
Distributions of the universe horizon distance and universe horizon volume were investigated in the light of five general cosmic models which were constructed in a previous study. Both distributions increase so slowly up to $t \approx 21.5444 \mathrm{Myr}$, then they start raising very fast up to $t \approx 60 \mathrm{Gyr}$. Afterwards, they increase again very slowly until $t \approx 124 \mathrm{Gyr}$. Distributions of mass of radiation, matter and dark energy within the horizon volume of the universe were also studied in the five general cosmic models. The masses of both radiation and matter decrease gradually with time while the mass of dark energy increases. The mass of radiation prevailed in the early universe up to $t \approx 34627.5$ - $55916.2 \mathrm{yr}$, where it becomes equal to the mass of matter. Then the mass of matter dominated until $t \approx 9.4525-10.0632 \mathrm{Gyr}$, where it becomes equal to the mass of dark energy. Thenceforward, the mass of dark energy prevails the universe. The cosmic space becomes approximately matter empty in the so far future of the universe.
\end{abstract}

\section{Keywords}

General Cosmic Models, Distribution of Mass and Energy

\section{Introduction}

In a previous study [1] the distribution of density parameters of radiation, matter and dark energy were investigated in details in five general cosmic models. Hence, it would be interesting to study the distributions of equivalent mass of radiation, mass of matter and equivalent mass of dark energy within the horizon volume of the universe in the general models.

Therefore, it is necessary to start this study by investigating the distributions of the horizon distance and horizon volume of the universe in the general models at different time intervals depending on the bases discussed in [2]. Description of methodology is given in Section 2 while algorithm would be illustrated in Section 3. Results 
and discussion are presented in Section 4. Conclusion is shown in Section 5.

\section{Methodology}

We have seen in [2] that the horizon distance and horizon volume of the universe at the present time are respectively

$$
\begin{gathered}
d_{h}\left(t_{o}\right)=\frac{c}{H_{o}} \int_{0}^{1} \frac{1}{a}\left[1-\Omega_{\Lambda, o}\left(1-a^{2}\right)+\Omega_{m, o}\left(\frac{1}{a}-1\right)+\Omega_{r, o}\left(\frac{1}{a^{2}}-1\right)\right]^{-\frac{1}{2}} \mathrm{~d} a . \\
V_{h}\left(t_{o}\right)=\frac{8 \pi}{3} d_{h}^{3}\left(t_{o}\right) .
\end{gathered}
$$

where $c, H_{o}, \Omega_{r, o}, \Omega_{m, o}, \Omega_{\Lambda, o}$ are all defined as in [1]. Thus the horizon distance of the universe at any given time is

$$
d_{h}(t)=\frac{c}{H_{o}} \int_{0}^{a} \frac{1}{a}\left[1-\Omega_{\Lambda, o}\left(1-a^{2}\right)+\Omega_{m, o}\left(\frac{1}{a}-1\right)+\Omega_{r, o}\left(\frac{1}{a^{2}}-1\right)\right]^{-\frac{1}{2}} \mathrm{~d} a .
$$

Consequently the change in the horizon distance of the universe in the time interval between two instants of scale factors $a_{1}, a_{2}$ is written as

$$
\Delta d_{h}(t)=\frac{c}{H_{o}} \int_{a_{1}}^{a_{2}} \frac{1}{a}\left[1-\Omega_{\Lambda, o}\left(1-a^{2}\right)+\Omega_{m, o}\left(\frac{1}{a}-1\right)+\Omega_{r, o}\left(\frac{1}{a^{2}}-1\right)\right]^{-\frac{1}{2}} \mathrm{~d} a .
$$

The horizon volume of the universe at any given time is

$$
V_{h}(t)=\frac{8 \pi}{3} d_{h}^{3}(t)
$$

It is also obvious from [2] that the total density of the universe is given by

$$
\rho(t)=\rho_{c, t} \Omega(t) .
$$

where

$$
\begin{gathered}
\Omega(t)=\rho_{c, t}\left(\Omega_{m, t}+\Omega_{r, t}+\Omega_{\Lambda, t}\right) . \\
\rho_{c, t}=\frac{3 H^{2}}{8 \pi G} . \\
\Omega_{m, t}=\frac{\rho_{m, t}}{\rho_{c, t}}=\left(\frac{H_{0}}{H}\right)^{2} \frac{\Omega_{m, o}}{a^{3}} . \\
\Omega_{r, t}=\frac{\rho_{r, t}}{\rho_{c, t}}=\left(\frac{H_{0}}{H}\right)^{2} \frac{\Omega_{r, o}}{a^{4}} . \\
\Omega_{\Lambda, t}=\frac{\rho_{\Lambda, t}}{\rho_{c, t}}=\left(\frac{H_{0}}{H}\right)^{2} \Omega_{\Lambda, o} . \\
H(t)=\frac{H_{o}}{a}\left[1-\Omega_{\Lambda, o}\left(1-a^{2}\right)+\Omega_{m, o}\left(\frac{1}{a}-1\right)+\Omega_{r, o}\left(\frac{1}{a^{2}}-1\right)\right]^{\frac{1}{2}} .
\end{gathered}
$$

Hence, the total mass within the horizon volume of the universe at any given time is expressed as

$$
M_{h}(t)=V_{h}(t) \rho(t) .
$$

The mass of matter $M_{m, t}$, the equivalent mass of radiation $M_{r, t}$ and the equivalent mass of dark energy 
$M_{\Lambda, t}$ within the horizon volume of the universe at any given time are given by

$$
\begin{gathered}
M_{m, t}=M_{h}(t) \frac{\rho_{m, t}}{\rho(t)} \\
M_{m, t}=M_{h}(t) \frac{\Omega_{m, t}}{\Omega(t)} . \\
M_{r, t}=M_{h}(t) \frac{\Omega_{r, t}}{\Omega(t)} . \\
M_{\Lambda, t}=M_{h}(t) \frac{\Omega_{\Lambda, t}}{\Omega(t)} .
\end{gathered}
$$

The cosmic time is given by Equation (16) in [1] as

$$
t=\frac{1}{H_{o}} \int_{0}^{a}\left[1-\Omega_{\Lambda, o}\left(1-a^{2}\right)+\Omega_{m, o}\left(\frac{1}{a}-1\right)+\Omega_{r, o}\left(\frac{1}{a^{2}}-1\right)\right]^{-\frac{1}{2}} \mathrm{~d} a .
$$

Thus the time interval between two instants with scale factors $a_{1}, a_{2}$ during the universe expansion is expressed as

$$
\Delta t=\frac{1}{H_{o}} \int_{a_{1}}^{a_{2}}\left[1-\Omega_{\Lambda, o}\left(1-a^{2}\right)+\Omega_{m, o}\left(\frac{1}{a}-1\right)+\Omega_{r, o}\left(\frac{1}{a^{2}}-1\right)\right]^{-\frac{1}{2}} \mathrm{~d} a .
$$

\section{Algorithm}

In determination of the distributions of $d_{h}(t), V_{h}(t), M_{h}(t), M_{m, t}, M_{r, t}$ and $M_{\Lambda, t}$ we use the following steps:

i) Set $t=0, d_{h}=0, K_{1}=1, K_{2}=2000$, then insert the value of $a_{\max }=0.098$ for $t \leq 0.5 \mathrm{Gyr}, a_{\max }=10$ for $0.5<t \leq 50 \mathrm{Gyr}$ and $a_{\max }=1000$ for $50<t \leq 124 \mathrm{Gyr}$.

ii) Compute $D A=\frac{a_{\max }}{D B L E\left(K_{2}\right)}$.

iii) Start general DO loop $I=K_{1}, K_{2}$ which includes the following sub steps:

iv) $a_{1}=D A(I-1), a_{2}=D A I$.

v) Calculate new value of cosmic time $t$ numerically using(16-b), where $t=t+\Delta t$.

vi) Determinate new value of the universe horizon distance $d_{h}$ numerically using (3-b) where, $d_{h}=d_{h}+\Delta d_{h}$.

vii) Obtain the corresponding values of $V_{h}, H, \rho_{c, t}, \Omega_{m, t}, \Omega_{r, t}, \Omega_{\Lambda, t}, \Omega(t), \rho(t), M_{h}(t), M_{m, t}, M_{r, t}, M_{\Lambda, t}$ using (4), (11), (7), (8), (9), (10), (6), (5), (12), (13), (14) and (15) respectively.

viii) Continue the general DO loop.

\section{Results and Discussion}

The distribution of the universe horizon distance in the general models up to $t=0.5 \mathrm{Gyr}$ is shown in Figure 1(a). The distributions of all models coincide on each other until $t \approx 21.5444 \mathrm{Myr}$. Then, the distributions of models A, B and C coincide on each other and get upper than the coincided distributions of models D and E. The universe horizon distance increases quite slowly with time in all general models up to $t \approx 21.5444 \mathrm{Myr}$, hence it stats raising very fast. The distribution of the universe horizon distance in the general models in the range $t=0.5$ - $50 \mathrm{Gyr}$ is illustrated in Figure 1 (b). The distributions of all models coincide on each other up to $t \approx 1.1111 \mathrm{Gyr}$. Afterwards, the distributions of models A, B and C coincide on each other and become higher than the coincided distributions of models $\mathrm{D}$ and $\mathrm{E}$. In all general models the universe horizon distance distributions increase very fast with time. The distribution of the universe horizon distance in the general models in the range $t=50-124 \mathrm{Gyr}$ is presented in Figure 1(c). The distributions of models A, B and C are close to each other and lie upper than the distributions of models $\mathrm{E}$ and $\mathrm{D}$. The increase in the universe horizon distance gets very small with increasing time in all general models. 


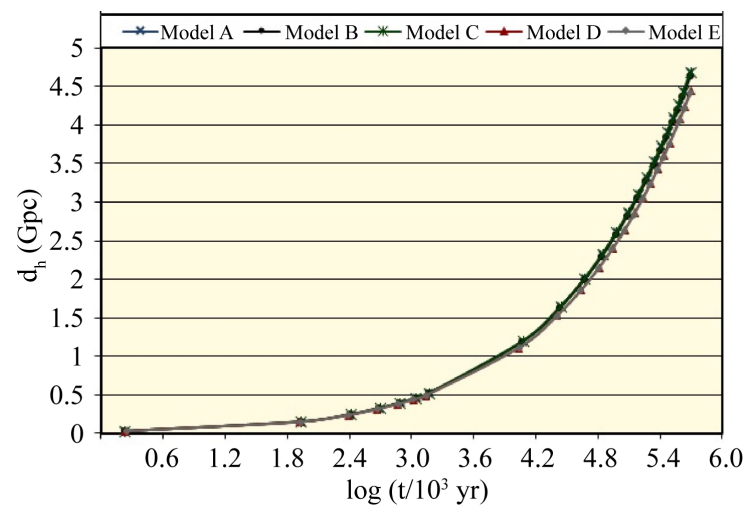

(a)

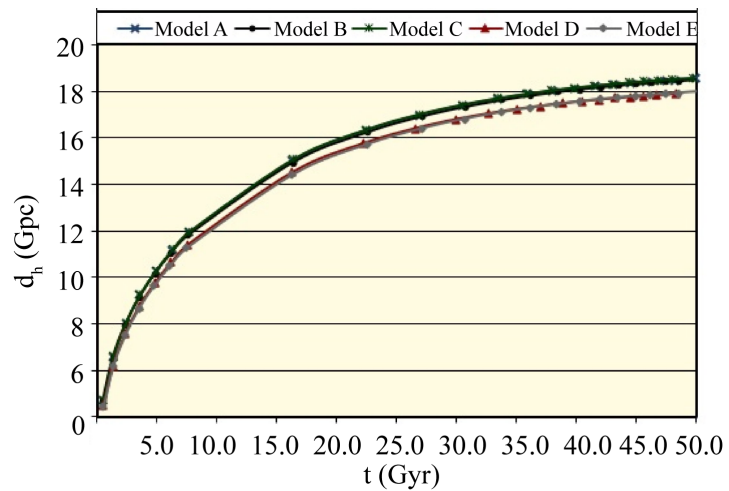

(b)

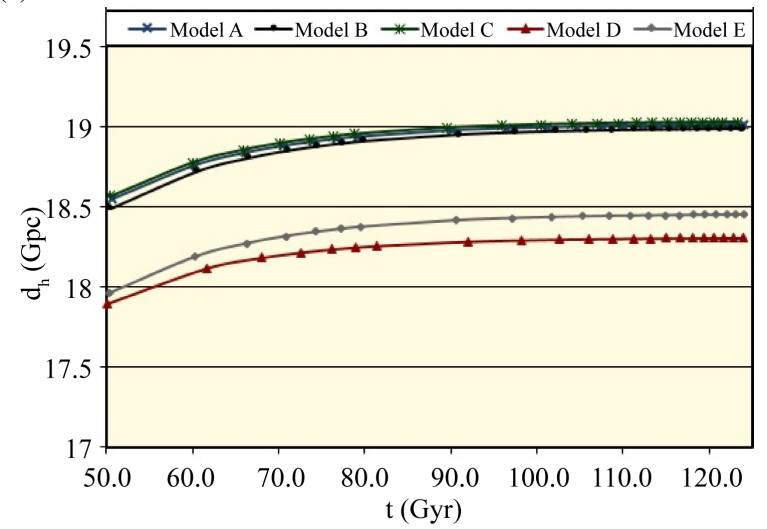

(c)

Figure 1. The distribution of the universe horizon distance in the general cosmic models (a) up to $t=0.5$ Gyr; (b) in the range $t=0.5-50 \mathrm{Gyr}$; (c) in the range $t=50-124 \mathrm{Gyr}$.

Table 1 shows the universe horizon distances in the general models at special times. These times are the time of radiation-matter mass equivalence $t_{r m}$, the time of matter-dark energy mass equivalence $t_{m \Lambda}$, the present time $t_{o}=13.7 \pm 0.2 \mathrm{Gyr}$ and the time $t_{n}=124 \mathrm{Gyr}$.

The results illustrated in Figures 1(a)-(c) are supported by those displayed in Figures 2(a)-(c) which show the distributions of the universe horizon volume in the general models in the ranges up to $t=0.5 \mathrm{Gyr}, t=0.5$ 50 Gyr and $t=50$ - 124 Gyr respectively. Table 2 presents the universe horizon volumes in the general models at the special times $t_{r m}, t_{m \Lambda}, t_{o}$ and $t_{n}$.

The distribution of mass and energy within the universe horizon volume of the universe in any general model up to $t=0.5 \mathrm{Gyr}$ is exhibited in Figure 3(a). The distributions of both radiation and matter decrease gradually with time and intersect at the time $t_{r m}=34627.5-55916.2 \mathrm{yr}$ as shown in Table 3. On the other hand, the distribution of dark energy increases gradually until it intersects with the radiation distribution at the time $t_{r \Lambda}=0.5166-0.5839 \mathrm{Gyr}$ as seen in Table 4. The distribution of total mass coincides with that of radiation up to $t \approx 5843.4141 \mathrm{yr}$. Afterwards, the two distributions diverge from each other. However, the distribution of the total mass coincides on the distribution of matter from the time $t \approx 857695.9 \mathrm{yr}$ onwards.

The distribution of mass and energy within the universe horizon volume of the universe in any general model in the range $t=0.5$ - $50 \mathrm{Gyr}$ is displayed in Figure 3(b). It is obvious that the distributions of matter and radiation decrease gradually with time and the former lies above the later. The distribution of dark energy increases with time and intersects with the distribution of matter at $t=9.4525-10.0632 \mathrm{Gyr}$ as illustrated in Table 5. The distribution of the total mass coincides on the distribution of the matter up to $t=4.5714$ Gyr , then they diverge from each other. Furthermore, the distribution of the total mass coincides on the distribution of the dark energy from $t=18.2857 \mathrm{Gyr}$ onwards. Masses of radiation, matter and dark energy within the universe horizon volume in the general models at the present time are illustrated in Table 6.

The distribution of mass and energy within the universe horizon volume in any general model in the range 
Table 1. Horizon distances of the universe in the general cosmic models at special times.

\begin{tabular}{|c|c|c|c|c|}
\hline Model & $d_{h}\left(t_{r m}\right) / \mathrm{Mpc}$ & $d_{h}\left(t_{m \Lambda}\right) / \mathrm{Gpc}$ & $d_{h}\left(t_{o}\right) / \mathrm{Gpc}$ & $d_{h}\left(t_{n}\right) / \mathrm{Gpc}$ \\
\hline A & 118.1520 & 12.7667 & 14.2969 & 19.0103 \\
\hline B & 112.1480 & 12.7586 & 14.1588 & 18.9849 \\
\hline $\mathrm{C}$ & 118.0740 & 12.7812 & 14.2780 & 19.0274 \\
\hline $\mathrm{D}$ & 98.0130 & 12.3367 & 13.8666 & 18.3062 \\
\hline $\mathrm{E}$ & 94.3170 & 12.4269 & 13.8070 & 18.4510 \\
\hline
\end{tabular}

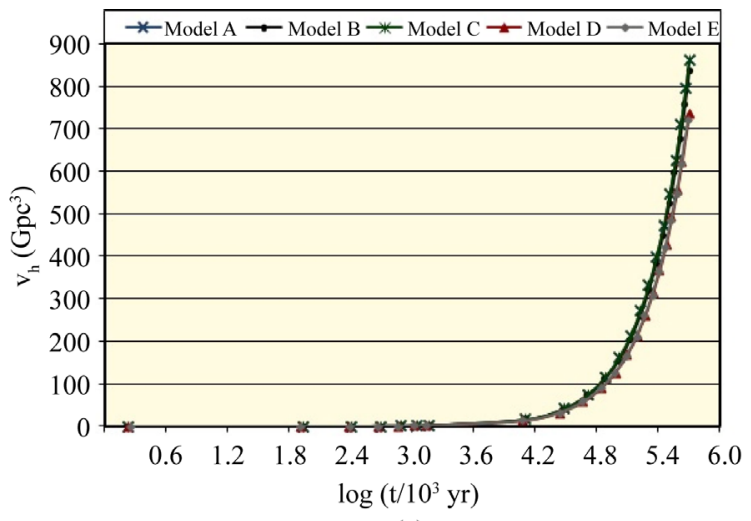

(a)

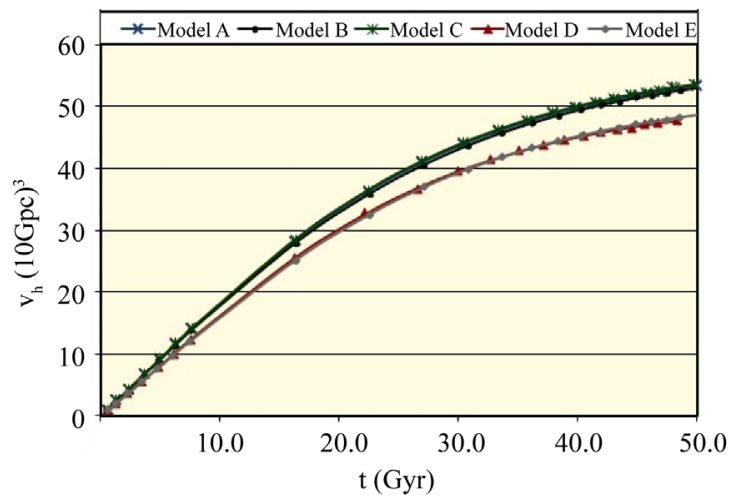

(b)

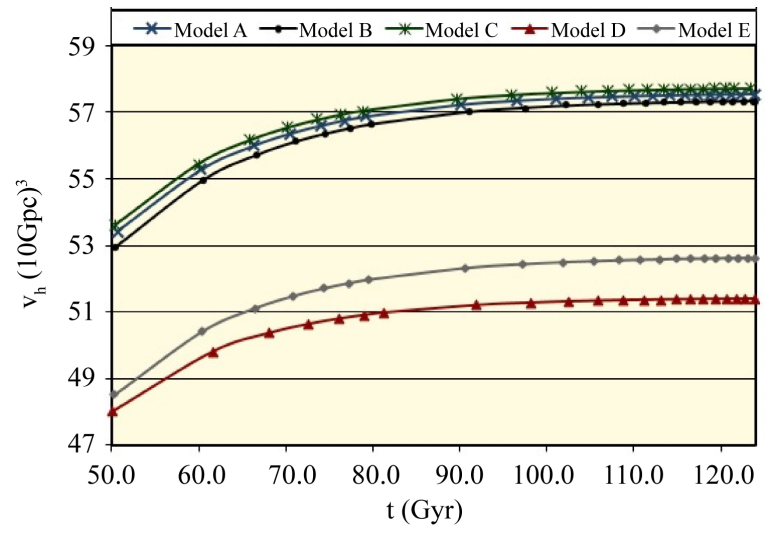

(c)

Figure 2. The distribution of the universe horizon volume in the general cosmic models (a) up to $t=0.5 \mathrm{Gyr}$; (b) in the range $t=0.5-50 \mathrm{Gyr}$; (c) in the range $t=50-124 \mathrm{Gyr}$.

Table 2. Horizon volumes of the universe in the general cosmic models at special times.

\begin{tabular}{ccccc}
\hline Model & $V_{h}\left(t_{r m}\right) / \mathrm{Mpc}^{3}$ & $V_{h}\left(t_{m \Lambda}\right) /(10 \mathrm{Gpc})^{3}$ & $V_{h}\left(t_{o}\right) /(10 \mathrm{Gpc})^{3}$ & $V_{h}\left(t_{n}\right) /(10 \mathrm{Gpc})^{3}$ \\
\hline A & 13820.0 & 17.4322 & 24.4819 & 57.5555 \\
B & 11820.0 & 17.3990 & 23.7792 & 57.3248 \\
C & 13790.0 & 17.4916 & 24.3849 & 57.7111 \\
D & 7890.0 & 15.7295 & 22.3372 & 51.3939 \\
E & 7030.0 & 16.0772 & 22.0504 & 52.6234
\end{tabular}




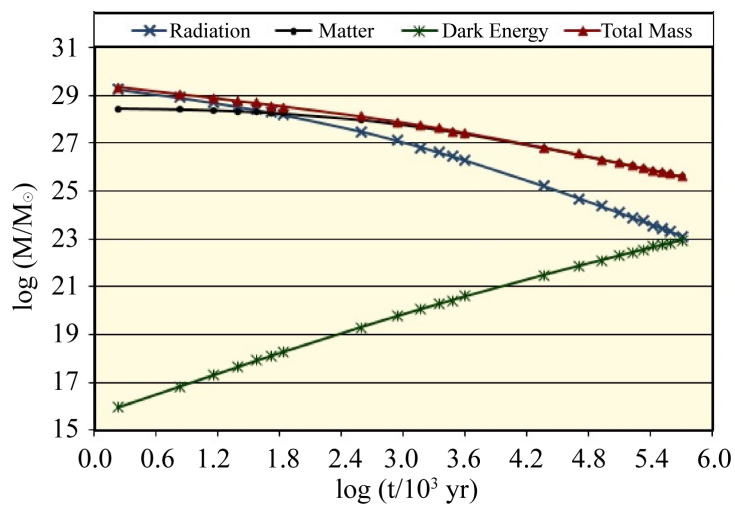

(a)

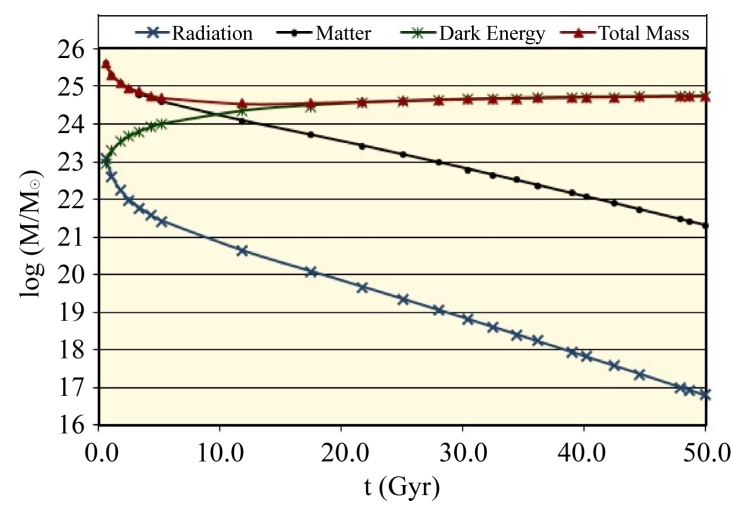

(b)

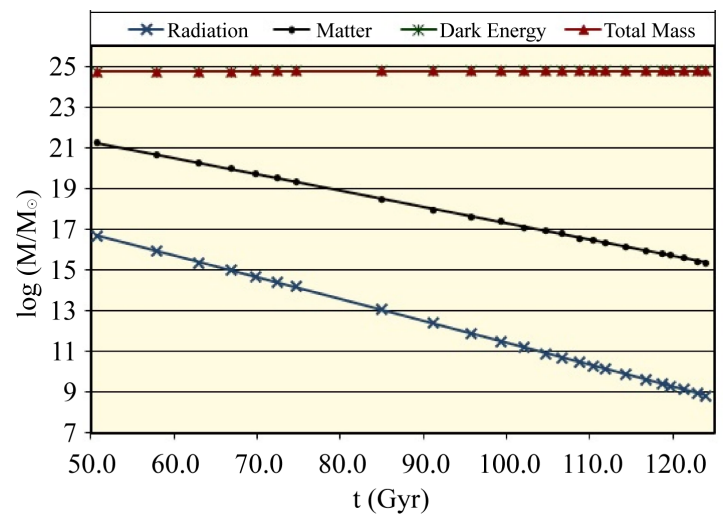

(c)

Figure 3. The distribution of mass and energy within the universe horizon volume in any general cosmic model (a) up to $t=$ $0.5 \mathrm{Gyr}$; (b) in the range $t=0.5-50 \mathrm{Gyr}$; (c) in the range $t=50-124 \mathrm{Gyr}$.

Table 3. Cosmic times at which $M_{r}(t)=M_{m}(t)$ within the universe horizon volume in the general cosmic models.

\begin{tabular}{cccc}
\hline Model & $t_{r m} / 10^{3}$ yr & $\log \left(M_{r m} / M_{\odot}\right)$ & $\log \left(M_{\Lambda} / M_{\odot}\right)$ \\
\hline A & 55.9162 & 28.2526 & 18.1498 \\
B & 49.6665 & 28.2876 & 18.0712 \\
C & 55.8428 & 28.2529 & 18.1562 \\
D & 38.4783 & 28.3338 & 17.9566 \\
E & 34.6275 & 28.3753 & 17.8515
\end{tabular}

Table 4. Cosmic times at which $M_{r}(t)=M_{\Lambda}(t)$ within the universe horizon volume in the general cosmic models.

\begin{tabular}{cccc}
\hline Model & $t_{r \Lambda} /$ Gyr & $\log \left(M_{r \Lambda} / M_{\odot}\right)$ & $\log \left(M_{m} / M_{\odot}\right)$ \\
\hline A & 0.5839 & 23.0153 & 25.5410 \\
B & 0.5736 & 22.9834 & 25.5375 \\
C & 0.5808 & 23.0202 & 25.5445 \\
D & 0.5166 & 22.9407 & 25.5350 \\
E & 0.5259 & 22.8906 & 25.5216 \\
\hline
\end{tabular}


$t=50-124 \mathrm{Gyr}$ is exhibited in Figure 3(c). Again the distribution of both matter mass and radiation mass decrease with time and the former is higher than the later. The distributions of dark energy mass and total mass coincide on each other. Masses of radiation, matter and dark energy within the universe horizon volume in the general models at $t_{n}$ are given in Table 7 .

Table 8 shows the equivalent number of the Coma-like clusters to the mass of matter within the universe horizon volume $N_{\text {СомА }}(t)$ in the general models at the special times $t_{r m}, t_{m \Lambda}, t_{o}$ and $t_{n}$. It is obvious that this content of matter strongly decreases with time such that the cosmic space becomes almost matter empty in the far future of the universe.

\section{Conclusion}

In this article distributions of the universe horizon distance and universe horizon volume were determined in the five general cosmic models which were established previously. The two distributions were found increasing slowly up to $t \approx 21.5444 \mathrm{Myr}$, hence they raise appreciably fast up to $t=60 \mathrm{Gyr}$, then they increase again so slowly until $t=124 \mathrm{Gyr}$. Distributions of mass of radiation, matter and dark energy within the universe horizon volume were also investigated in the five general models. The masses of radiation and matter are decreasing with time although the mass of dark energy is increasing. The mass of radiation was dominant in the early

Table 5. Cosmic times at which $M_{m}(t)=M_{\Lambda}(t)$ within the universe horizon volume in the general cosmic models.

\begin{tabular}{cccc}
\hline Model & $t_{m \Lambda} /$ Gyr & $\log \left(M_{m \Lambda} / M_{\odot}\right)$ & $\log \left(M_{r} / M_{\odot}\right)$ \\
\hline A & 9.4650 & 24.2507 & 20.8831 \\
B & 9.6930 & 24.2393 & 20.8339 \\
C & 9.4525 & 24.2594 & 20.8937 \\
D & 9.6289 & 24.2564 & 20.7974 \\
E & 10.0632 & 24.2109 & 20.7030 \\
\hline
\end{tabular}

Table 6. Masses of radiation, matter and dark energy within the universe horizon volume in the general cosmic models at $t=t_{0}$.

\begin{tabular}{cccc}
\hline Model & $\log \left(M_{r} / M_{\odot}\right)$ & $\log \left(M_{m} / M_{\odot}\right)$ & $\log \left(M_{\Lambda} / M_{\odot}\right)$ \\
A & 20.4531 & 23.9625 & 24.3973 \\
B & 20.4324 & 23.9683 & 24.3785 \\
C & 20.4570 & 23.9663 & 24.4086 \\
D & 20.4035 & 23.9954 & 24.3981 \\
E & 20.3684 & 23.9921 & 24.3328 \\
\hline
\end{tabular}

Table 7. Masses of radiation, matter and dark energy within the universe horizon volume in the general cosmic models at $t=t_{n}$.

\begin{tabular}{cccc}
\hline Model & $\log \left(M_{r} / M_{\odot}\right)$ & $\log \left(M_{m} / M_{\odot}\right)$ & $\log \left(M_{\Lambda} / M_{\odot}\right)$ \\
A & 8.8400 & 15.3480 & 24.7694 \\
B & 8.9797 & 15.4781 & 24.7571 \\
C & 8.7546 & 15.2847 & 24.7779 \\
D & 8.1326 & 14.8863 & 24.7706 \\
E & 8.8615 & 15.4585 & 24.7258 \\
\hline
\end{tabular}


Table 8. Equivalent number of the Coma-like clusters to the mass of matter within the universe horizon volume in the general cosmic models at special times.

\begin{tabular}{ccccc}
\hline Model & $t_{r m}$ & $t_{m \Lambda}$ & $t_{o}$ & $t_{n}$ \\
\hline A & $8.9448 \times 10^{12}$ & $8.9057 \times 10^{8}$ & $4.5864 \times 10^{8}$ & 1.1142 \\
B & $9.6955 \times 10^{12}$ & $8.6750 \times 10^{8}$ & $4.6480 \times 10^{8}$ & 1.5034 \\
C & $8.9510 \times 10^{12}$ & $9.0859 \times 10^{8}$ & $4.6267 \times 10^{8}$ & 0.9631 \\
D & $10.7838 \times 10^{12}$ & $9.0234 \times 10^{8}$ & $4.9473 \times 10^{8}$ & 0.3848 \\
E & $11.8651 \times 10^{12}$ & $8.1259 \times 10^{8}$ & $4.9099 \times 10^{8}$ & 1.4370 \\
\hline
\end{tabular}

universe up to $t=34627.5$ - $55916.2 \mathrm{yr}$, where it becomes equivalent to the mass of matter. Afterwards, the mass of matter prevailed until $t=9.4525-10.0632 \mathrm{Gyr}$, where it becomes equal to the mass of dark energy. From this time onwards the mass of dark energy dominates the universe. The cosmic space gets approximately matter empty in the very remote future of the universe.

\section{References}

[1] Bukhari, F.A. (2013) Five General Cosmic Models. Journal of King Abdulaziz University: Science, 25.

[2] Bukhari, F.A. (2013) Cosmological Distances in Five General Cosmic Models. International Journal of Astronomy and Astrophysics, 3, 183-188. 